\title{
The modulation of the gamma-ray emission from the binary LS 5039
}

\author{
G. Dubus, B. Cerutti, and G. Henri \\ Laboratoire d'Astrophysique de Grenoble, UMR 5571 CNRS, Université Joseph Fourier, BP 53, 38041 Grenoble, France
}

Received 12 July 2007 / Accepted 2 October 2007

\begin{abstract}
Context. Gamma-ray binaries have been established as a new class of sources of very high energy (VHE, $>100 \mathrm{GeV}$ ) photons. These binaries are composed of a massive star and a compact object. The gamma rays are probably produced by inverse Compton scattering of the stellar light by VHE electrons accelerated in the vicinity of the compact object. The VHE emission from LS 5039 displays an orbital modulation.

Aims. The inverse Compton spectrum depends on the angle between the incoming and outgoing photons in the rest frame of the electron. Since the angle at which an observer sees the star and electrons changes with the orbit, a phase dependence of the spectrum is expected.

Methods. A procedure for computing anisotropic inverse Compton emission is explained and applied to the case of LS 5039. The spectrum is calculated assuming the continuous injection of electrons close to the compact object: the shape of the steady-state distribution depends on the injected power law and on the magnetic-field's intensity.

Results. Compared to the isotropic approximation, anisotropic scattering produces harder and fainter emission at inferior conjunction, crucially at a time when the attenuation due to pair production of the VHE gamma-rays on star light is minimum. The computed light curve and spectra are very good fits to the HESS and EGRET observations, except at phases of maximum attenuation where pair cascade emission may be significant for HESS. Detailed predictions are made for a modulation in the GLAST energy range. The magnetic field intensity at periastron is $0.8 \pm 0.2 \mathrm{G}$.

Conclusions. The anisotropy in inverse Compton scattering plays a major role in LS 5039. A simple model reproduces the observations, constraining the magnetic field intensity and injection spectrum. The comparison with observations, the derived magnetic field intensity, injection energy, and slope together suggest emission from a rotation-powered pulsar wind nebula. These results confirm gamma-ray binaries as promising sources for studying the environment of pulsars on small scales.
\end{abstract}

Key words. radiation mechanisms: non-thermal - stars: individual (LS 5039) - gamma rays: theory - X-rays: binaries

\section{Introduction}

Gamma-ray binaries have been established in the past couple of years as a new class of sources of very high energy (VHE, $>100 \mathrm{GeV}$ ) photons. They are characterised by high gamma-ray luminosity above an $\mathrm{MeV}$, at the level of or exceeding their X-ray luminosity. At present, all three such known systems (LS 5039, PSR B1259-63, and LSI $+61^{\circ} 303$, recently possibly joined by Cyg X-1) comprise a massive star (Aharonian et al. 2005a,b; Albert et al. 2006, 2007). The compact object in PSR B1259-63 is a $48 \mathrm{~ms}$, young radio pulsar. The VHE emission arises from the interaction of the relativistic wind from this pulsar, extracting rotational energy from the neutron star, with the stellar wind from its companion (Tavani et al. 1994). Particles gain energy at the shock between the winds, resulting in a small-scale pulsar wind nebula (Maraschi \& Treves 1981). The particles radiate their energy away as they are entrained in the shocked flow, forming a comet-like trail of emission behind the pulsar (Dubus 2006b).

The nature of the compact object and origin of the VHE emission remains controversial in LS 5039 and LSI $+61^{\circ} 303$, although recent observations indicate that the radio emission of LSI $+61^{\circ} 303$ behaves like the comet tail expected in the pulsar scenario (Dhawan et al. 2006). Alternatively, the VHE emission could originate from particles accelerated in a relativistic jet, the energy source being accretion onto a black hole or a neutron star (Dermer \& Böttcher 2006; Paredes et al. 2006). The rationale is that there is evidence of particle acceleration in the jets of microquasars and active galactic nuclei. However, hard evidence of accretion occurring in either LS 5039 or LSI $+61^{\circ} 303$ has been hard to come by (e.g. Martocchia et al. 2005), and the similarities between the three systems (and differences with the usual microquasars) do not argue in favour of the accretion/ejection scenario (Dubus 2006b).

Regardless of the actual powering mechanism, some particles must be accelerated to high energies to generate the VHE gamma-rays. If these particles are leptons, the only viable gamma-ray radiation mechanism is inverse Compton scattering on the stellar photons. The massive stars in gamma-ray binaries have effective temperatures of several tens of thousand $\mathrm{K}$ and radii of about $10 R_{\odot}$, yielding luminosities of the order of $10^{39} \mathrm{erg} \mathrm{s}^{-1}$. This provides a huge density of stellar photons in the UV band that VHE leptons may up-scatter, much greater than any other possible source of target photons (e.g. synchrotron or bremsstrahlung emission).

The emitted VHE photons also have enough energy to produce $e^{+} e^{-}$pairs with the UV stellar photons. Most of the VHE flux may therefore be lost to the observer if the source is behind the star and VHE photons have to travel through the stellar light. 
Gamma-ray attenuation has been shown to lead to a modulation of the VHE flux with minimum absorption (maximum) at inferior (superior) conjunction (Böttcher \& Dermer 2005; Dubus 2006a).

HESS observations have indeed shown a stable modulation of the VHE flux from LS 5039 on the orbital period with a maximum around inferior conjunction. This suggests that attenuation plays a role and that the source of VHE gamma-rays cannot be more than about an AU from the binary (or attenuation would be too weak to modulate the flux). However, a non-zero flux is detected at superior conjunction where a large attenuation is expected, possibly because of pair cascading. Moreover, the spectral changes that are reported do not fit with an interpretation based on pure attenuation of a constant VHE source spectrum (Aharonian et al. 2006).

Inverse Compton scattering also has a well-known dependence on the angle $\Theta$ between incoming and outgoing photons. The photon flux from the star being anisotropic, the resulting inverse Compton emission will depend on the angle at which it is observed. Hence, a phase-dependent VHE spectrum will be observed even if the distribution of particles is isotropic and remains constant throughout. This effect has previously been investigated for the Crab pulsar by Bogovalov \& Aharonian (2000), and in PSR B1259-63 by Ball \& Kirk (2000). Ball \& Kirk calculated the radiative drag on the unshocked pulsar wind from scattering of stellar light, using results from Ho \& Epstein (1989). The drag produces a Compton gamma-ray line with a strong dependence on viewing angle.

This work purports to explain the HESS observations of LS 5039 using a combination of anisotropic inverse Compton scattering and attenuation in the simplest way possible. The aim is to constrain the underlying particle distribution and/or powering mechanism. The main equations governing anisotropic Compton scattering in the context of gamma-ray binaries are derived in Sect. 2, which also discusses the principal characteristics to expect. The application to the case of LS 5039 is presented in Sect. 3. The light curve and spectra observed by the HESS collaboration are reproduced by a model taking into account the photon field anisotropy and the attenuation due to pair creation. Section 4 concludes on the origin of the VHE emission from this system.

\section{Anisotropic Compton scattering}

Quantities in the electron rest frame are primed and quantities in the observer frame are left unprimed. The electron energy is $\gamma_{\mathrm{e}} m_{\mathrm{e}} c^{2}$, the energy of the incoming (stellar) photon is $\epsilon_{0}$ and the outgoing photon energy is $\epsilon_{1}$. These quantities are related in the electron rest frame by the standard

$$
\epsilon_{1}^{\prime}=\frac{\epsilon_{0}^{\prime}}{1+\frac{\epsilon_{0}^{\prime}}{m_{\mathrm{e}} c^{2}}\left(1-\cos \Theta^{\prime}\right)}
$$

with $\Theta^{\prime}$ the angle between the incoming and outgoing photons. The incoming and outgoing photon energies are equal $\epsilon_{1}^{\prime}=\epsilon_{0}^{\prime}$ in the Thomson scattering approximation $\epsilon_{0}^{\prime} \ll m_{\mathrm{e}} c^{2}$, or $\epsilon_{0} \ll$ $m_{\mathrm{e}} c^{2} /\left[\gamma_{\mathrm{e}}\left(1-\beta \cos \theta_{0}\right)\right]$ when expressed in the observer frame $\left(\theta_{0}\right.$ is the photon angle with respect to the electron direction of motion). Scattering is also Thomson-like even if $\gamma_{\mathrm{e}} \epsilon_{0}>m_{\mathrm{e}} c^{2}$ when the incoming and outgoing photon have almost the same direction $\left(\Theta^{\prime} \ll\left(2 m_{\mathrm{e}} c^{2} / \epsilon_{0}^{\prime}\right)^{1 / 2}\right)$. In the observer frame there is also an angle $\theta_{\text {crit }}$ below which scattering will be Thomson-like. This angle is defined by

$\cos \theta_{\text {crit }} \gtrsim \frac{1}{\beta}\left(1-\frac{m_{\mathrm{e}} c^{2}}{\gamma_{\mathrm{e}} \epsilon_{0}}\right) ;$

i.e. $\theta_{\text {crit }} \lesssim 60^{\circ}$ for $\gamma \epsilon_{0}=1 \mathrm{MeV}$. The cross-section in the electron rest frame is

$\frac{\mathrm{d} \sigma}{\mathrm{d} \epsilon_{1}^{\prime} \mathrm{d} \Omega_{1}^{\prime}}\left(\epsilon_{0}^{\prime}, \epsilon_{1}^{\prime}, \Theta^{\prime}\right)=\frac{r_{\mathrm{e}}^{2}}{2}\left(\frac{\epsilon_{1}^{\prime}}{\epsilon_{0}^{\prime}}\right)^{2}\left(\frac{\epsilon_{1}^{\prime}}{\epsilon_{0}^{\prime}}+\frac{\epsilon_{0}^{\prime}}{\epsilon_{1}^{\prime}}-\sin ^{2} \Theta^{\prime}\right)$

where $r_{\mathrm{e}}$ is the classical electron radius and the photon energies $\epsilon_{0,1}^{\prime}$ are related through Eq. (1) (note that the dirac relating the two energies has been dropped in Eq. (3) above for legibility see also Eq. (A.1)).

\subsection{Monoenergetic beam}

It is worthwhile considering first the simple case of a monoenergetic beam of photons scattering off a single electron. The main steps are listed below and a detailed derivation may be found in Fargion et al. (1997).

In the observer frame, the incoming photon density (in $\mathrm{sr}^{-1} \mathrm{erg}^{-1}$ ), normalised to the (constant) total photon density $n_{0}$ (in photons $\mathrm{cm}^{-3}$ ), is

$$
\frac{\mathrm{d} n}{\mathrm{~d} \epsilon \mathrm{d} \Omega}=\delta\left(\epsilon-\epsilon_{0}\right) \delta\left(\cos \theta-\cos \theta_{0}\right) \delta\left(\phi-\phi_{0}\right)
$$

with $\delta$ the Dirac function. The frame origin is at the location of the electron (the frame orientation is arbitrary). The photon density in the electron frame is found by using the invariance of $\mathrm{d} n / \mathrm{d} \epsilon$ (Blumenthal \& Gould 1970).

The fraction of photons scattered per unit time, energy, and solid angle in the electron frame is then given by (Jones 1968; Blumenthal \& Gould 1970)

$\frac{\mathrm{d} N^{\prime}}{\mathrm{d} t^{\prime} \mathrm{d} \epsilon_{1}^{\prime} \mathrm{d} \Omega_{1}^{\prime}}=\iint c \frac{\mathrm{d} \sigma}{\mathrm{d} \epsilon_{1}^{\prime} \mathrm{d} \Omega_{1}^{\prime}} \frac{\mathrm{d} n^{\prime}}{\mathrm{d} \epsilon^{\prime} \mathrm{d} \Omega^{\prime}} \mathrm{d} \Omega^{\prime} \mathrm{d} \epsilon^{\prime}$

which can be transformed to the observer frame using that the number of photons is invariant:

$\frac{\mathrm{d} N}{\mathrm{~d} t \mathrm{~d} \epsilon_{1} \mathrm{~d} \Omega_{1}}=\frac{\mathrm{d} N^{\prime}}{\mathrm{d} t^{\prime} \mathrm{d} \epsilon_{1}^{\prime} \mathrm{d} \Omega_{1}^{\prime}} \frac{\mathrm{d} t^{\prime}}{\mathrm{d} t} \frac{\mathrm{d} \epsilon_{1}^{\prime}}{\mathrm{d} \epsilon_{1}} \frac{\mathrm{d} \Omega_{1}^{\prime}}{\mathrm{d} \Omega_{1}}$.

Here, $\Omega_{1}^{\prime}$ denotes the solid angle into which the outgoing photon is emitted and $\cos \Theta^{\prime}=\cos \theta^{\prime} \cos \theta_{1}^{\prime}+\sin \theta^{\prime} \sin \theta_{1}^{\prime} \cos \left(\phi_{1}^{\prime}-\phi^{\prime}\right)$. Defining the polar angles $\theta_{0,1}$ with respect to the direction of electron motion, the resulting differential photon spectrum is a function of $\gamma_{\mathrm{e}}, \theta_{0}, \phi_{0}, \epsilon_{0}, \theta_{1}, \phi_{1}$ and $\epsilon_{1}$. The integration gives a rather unwieldy expression that can be found in the Appendix (Eq. (A.2)).

In the Thomson regime $\left(\epsilon_{0}^{\prime} \ll m_{\mathrm{e}} c^{2}\right)$, the outgoing photon energy is unequivocally related to the incoming photon energy since $\epsilon_{1}^{\prime}=\epsilon_{0}^{\prime}$. A unique photon energy corresponds to each polar angle $\theta_{1}$. In the general regime there is also a dependence on the azimuth (see Appendix). Staying in the Thomson regime, the total spectrum emitted by an electron follows from the integration over $\mathrm{d} \Omega_{1}^{\prime}$ of Eq. (5) and is (Fargion et al. 1997)

$\frac{\mathrm{d} N}{\mathrm{~d} t \mathrm{~d} \epsilon_{1}}=\frac{\pi r_{\mathrm{e}}^{2} c}{2 \beta \gamma_{\mathrm{e}}^{2} \epsilon_{0}}\left[3-\mu_{0}^{\prime 2}+\frac{1}{\beta^{2}}\left(3 \mu_{0}^{\prime 2}-1\right)\left(\frac{\epsilon_{1}}{\gamma_{\mathrm{e}} \epsilon_{0}^{\prime}}-1\right)^{2}\right]$

where $\mu_{0}^{\prime}=\cos \theta_{0}^{\prime}$ and $\epsilon_{1}$ varies between $\epsilon_{0}\left(1-\beta \mu_{0}\right) /(1 \pm \beta)$. This expression shows how the emitted spectrum depends upon the angle $\theta_{0}$ between the monochromatic point source and the direction of motion of the electron. A more general expression is given in the Appendix (Eq. (A.6)). 


\subsection{Kernel for spectral calculations}

The monochromatic, single-photon result can be used as a kernel to integrate over general electron and incoming photon distributions. The total spectrum in photons $\mathrm{s}^{-1} \mathrm{erg}^{-1} \mathrm{sr}^{-1}$ is then given by

$$
\frac{\mathrm{d} N_{\text {tot }}}{\mathrm{d} t \mathrm{~d} \epsilon_{1} \mathrm{~d} \Omega_{1}}=\iiint \int \frac{\mathrm{d} N}{\mathrm{~d} t \mathrm{~d} \epsilon_{1} \mathrm{~d} \Omega_{1}} n_{0} \mathrm{~d} \Omega_{0} \mathrm{~d} \epsilon_{0} \frac{\mathrm{d} n_{\mathrm{e}}}{\mathrm{d} \gamma_{\mathrm{e}} \mathrm{d} \Omega_{\mathrm{e}}} \mathrm{d} \Omega_{\mathrm{e}} \mathrm{d} \gamma_{\mathrm{e}}
$$

where the evaluation of the kernel must take into account the changes in electron direction with respect to the given direction. However, this expression can be simplified.

The electron energy must be very high $\gamma_{\mathrm{e}} \gg 1$ in order to emit VHE photons. The emission is strongly forward-boosted in the direction of the electron motion by relativistic aberrations. An observer looking at the inverse Compton emission from an isotropic cloud of relativistic electrons essentially sees only the emission emitted by those electrons moving within an angle $1 / \gamma_{\mathrm{e}}$ from the line-of-sight (see e.g. Ball \& Kirk 2000). Their emission is almost entirely focused into the line-of-sight. Photons emitted slightly away from the line-of-sight and included in the integration compensate to order $1 / \gamma_{\mathrm{e}}$ for the emission from electrons moving at larger angles. Therefore, to a good approximation,

$\left.\left.\int \frac{\mathrm{d} N}{\mathrm{~d} t \mathrm{~d} \epsilon_{1} \mathrm{~d} \Omega_{1}} \frac{\mathrm{d} n_{\mathrm{e}}}{\mathrm{d} \gamma_{\mathrm{e}} \mathrm{d} \Omega_{\mathrm{e}}} \mathrm{d} \Omega_{\mathrm{e}} \approx \frac{\mathrm{d} N}{\mathrm{~d} t \mathrm{~d} \epsilon_{1}}\right|_{\alpha} \frac{\mathrm{d} n_{\mathrm{e}}}{\mathrm{d} \gamma_{\mathrm{e}} \mathrm{d} \Omega_{\mathrm{e}}}\right|_{\Omega_{1}}$,

and the spectrum will be given by

$$
\frac{\mathrm{d} N_{\text {tot }}}{\mathrm{d} t \mathrm{~d} \epsilon_{1} \mathrm{~d} \Omega_{1}}=\left.\left.\iiint n_{0} \mathrm{~d} \Omega_{0} \mathrm{~d} \epsilon_{0} \frac{\mathrm{d} N}{\mathrm{~d} t \mathrm{~d} \epsilon_{1}}\right|_{\alpha} \frac{\mathrm{d} n_{\mathrm{e}}}{\mathrm{d} \gamma_{\mathrm{e}} \mathrm{d} \Omega_{\mathrm{e}}}\right|_{\Omega_{1}} \mathrm{~d} \gamma_{\mathrm{e}}
$$

where the kernel is given by Eq. (7) or Eq. (A.6), evaluated at the angle $\alpha$ between the point-like photon source, the electron cloud, and the observer. If $\boldsymbol{e}_{\mathrm{obs}}$ is a unit vector from the electron cloud to the observer and $\boldsymbol{e}_{0}$ is a unit vector from the photon source to the electron cloud, expressed using $\theta_{0}$ and $\phi_{0}$, then $\mu_{\alpha} \equiv \cos \alpha=\boldsymbol{e}_{0} . \boldsymbol{e}_{\mathrm{obs}}$.

For scattering on an isotropic distribution of photons, $\boldsymbol{e}_{\mathrm{obs}}$ can be arbitrarily oriented so that $\alpha=\theta_{0}$. For a blackbody of temperature $T_{\star}$,

$$
n_{0} \mathrm{~d} \Omega_{0}=\frac{2}{h^{3} c^{3}} \frac{\epsilon_{0}^{2}}{\exp \left(\epsilon_{0} / k T_{\star}\right)-1} \mathrm{~d} \Omega_{0} \equiv f_{0} \mathrm{~d} \Omega_{0} .
$$

For a point-like star of radius $R_{\star}$ at a distance $d_{\star}$ from the electrons, $\boldsymbol{e}_{\text {obs }}$ can be defined on the plane containing the three locations so that, again, $\alpha=\theta_{0}$. The photon distribution is

$n_{0} \mathrm{~d} \Omega_{0}=\pi\left(\frac{R_{\star}}{d_{\star}}\right)^{2} f_{0} \delta\left(\mu_{0}-\mu_{\psi}\right) \delta\left(\phi_{0}\right) \mathrm{d} \Omega_{0}$

with $f_{0}$ as defined in the previous equation and where $\psi$ is the angle between the star centre, the cloud and the observer. The integral on $\Omega_{0}$ is direct, so the kernel only needs be numerically integrated on $\epsilon_{0}$ and $\gamma_{\mathrm{e}}$. Finally, for a star of finite size, the integration element is

$n_{0} \mathrm{~d} \Omega_{0}=f_{0} \cos \theta_{0} \mathrm{~d} \Omega_{0}, \quad \phi_{0} \in[0,2 \pi], \sin \theta_{0} \in\left[0, R_{\star} / d_{\star}\right]$

and $\mu_{\alpha}=\cos \psi \cos \mu_{0}+\sin \psi \sin \mu_{0} \cos \phi_{0}$. This requires a quadruple numerical integral.

The electron distribution will be assumed to be isotropic in the following so that the expression in Eq. (9) is a function $f_{\mathrm{e}}$ of $\gamma_{\mathrm{e}}$ only and $\int f_{\mathrm{e}} \mathrm{d} \gamma_{\mathrm{e}}$ gives the total number of electrons per steradian.

\subsection{Anisotropic scattering of stellar photons}

Figure 1 shows example calculations of the inverse Compton spectrum from a distribution of electrons scattering photons emitted by a star, as seen from different viewing angles. The incoming photons have a blackbody distribution, and the electrons have a power-law distribution $\mathrm{d} n_{\mathrm{e}}=\gamma_{\mathrm{e}}^{-2} \mathrm{~d} \gamma_{\mathrm{e}}$. The viewing angle $\psi$ is defined as the angle between the star, electron cloud and observer $\left(\cos \psi=\boldsymbol{e}_{\star} . \boldsymbol{e}_{\mathrm{obs}}\right.$ with $\boldsymbol{e}_{\star}$ a unit vector from the star centre to the cloud). Two cases are shown: one corresponding to scattering in the Thomson regime and one for the Klein-Nishina regime. For each regime, we compare the results obtained in the point source approximation and the results taking the finite size of the star into account.

When scattering occurs in the Thomson regime $\left(\epsilon_{0}^{\prime} \ll m_{\mathrm{e}} c^{2}\right)$, the maximum energy $\gamma_{\mathrm{e}}^{2} \epsilon_{0}(1+\beta)(1-\beta \cos \psi)$ decreases with decreasing viewing angle $\psi$, i.e. when the electrons move in front of the star as seen by the observer (left panel of Fig. 1). This is to be expected, because the electrons are then forward-scattering radiation that is less energetic in their rest-frame than in the head-on case because of the $1-\beta \cos \psi$ term in the Lorentz transform. The other effect is a lower rate of emission for low $\psi$ (as can be directly deduced from Eq. (6) and seen in the left panel of Fig. 1). This is also due to the decrease in the density of incoming photons in the electron rest frame when both particles move in the same direction. Scattering is more likely to occur when the particles collide head-on (e.g. Sazonov \& Sunyaev 2000).

These effects are pronounced in the point-source approximation and are diluted when taking the finite size of the star into account (see dashed lines compared to full lines in Fig. 1). With a star of finite size, electrons see incoming photons from a variety of angles, which contributes to raising the seed photon density in the electron rest frame when $\psi=0$ (and to slightly decreasing it at $\psi=\pi$ ). Because the density is tied to $1-\beta \cos \psi$, this suggests a simple rule-of-thumb, corroborated by numerical investigations: the effect of the finite star-size should be taken into account when $\sin \psi \lesssim R_{\star} / d_{\star}$ but can otherwise be neglected. If the observer is within the cone defined by the star with the electrons at apex, then the density of photons seen by the electrons moving towards the observer will be significantly greater than in the point-source case. Outside of this cone, the difference with a point-source approximation is minor. In Fig. 1, the star angular size seen by the electrons is $30^{\circ}$ (defining the cone opening angle) and the point-source approximation is indeed acceptable for $\psi>30^{\circ}$.

When scattering occurs in the Klein-Nishina regime $\left(\epsilon_{0}^{\prime}>\right.$ $m_{\mathrm{e}} c^{2}$ ), the maximum energy is almost constant at $\gamma_{\max } m_{\mathrm{e}} c^{2}$ regardless of viewing angle. For large viewing angles, the spectrum is soft due to the decrease in cross-section in this regime, just as in the isotropic case. At small viewing angles, the seed photon energy in the rest frame of the electron is lower than in the head-on case because of the angle dependence in the relativistic boost, as described above for the Thomson regime. Moreover, since the limit between Thomson and Klein-Nishina regimes is at $\epsilon_{0} \gamma_{\mathrm{e}}(1-\beta \cos \psi) \approx m_{\mathrm{e}} c^{2}$, scattering can reach back to the Thomson regime for small enough viewing angles, regardless of the electron energy (see Eq. (2) in Sect. 2). There are two consequences. First, the amplitude of the variations with viewing angle is smaller than in the Thomson regime, because at small $\psi$ the decrease in photon density is compensated for by the larger cross-section. Second, since there is no drop in cross-section at small $\psi$, there can be a significant hardening of the spectrum compared to the spectrum at larger $\psi$ (right panel of Fig. 1). These spectral effects may play an important role in modelling 

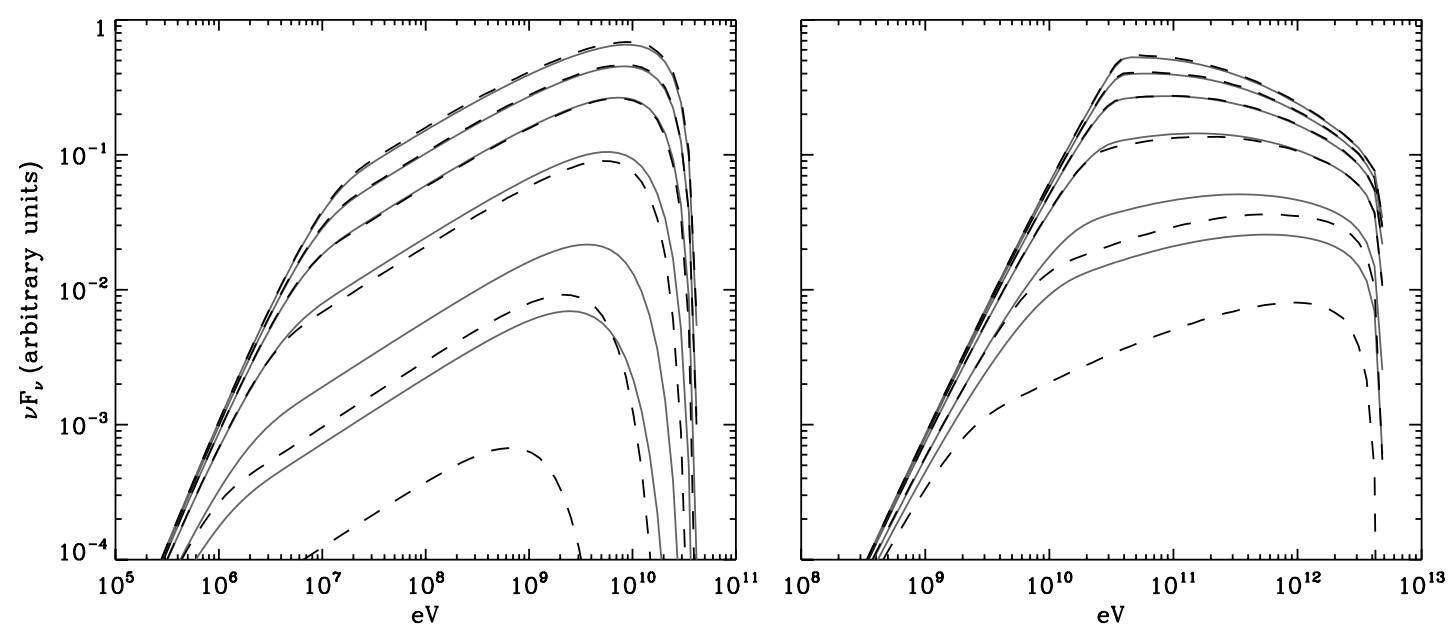

Fig. 1. Dependence of the viewing angle on the inverse Compton spectrum. The source of photons is a star with $k T=1 \mathrm{eV}$. The electron cloud is situated at a distance $d=2 R_{\star}$. The electrons are distributed according to a power law $\mathrm{d} n_{\mathrm{e}}=\gamma_{\mathrm{e}}^{-2} \mathrm{~d} \gamma_{\mathrm{e}}$. The left panel shows the variation of the spectrum with angle when the interaction occurs in the Thomson regime (electron energy range $10^{3}<\gamma_{\mathrm{e}}<10^{5}$ ). In the right panel the interaction occurs in the Klein-Nishina regime (electron range $\left.10^{5}<\gamma_{\mathrm{e}}<10^{7}\right)$. In each panel, the spectrum is shown at viewing angles $\psi=15^{\circ}($ bottom), $30^{\circ}, 60^{\circ}, 90^{\circ}, 120^{\circ}$ and $180^{\circ}$ (top). The observer sees the electron cloud in front of the seed photon source when the angle is small. Solid lines are calculated taking the finite star size into account (Eq. (13)); dashed lines correspond to the point source approximation (Eq. (12)).

the emission from gamma-ray binaries, for which scattering occurs mostly in the Klein-Nishina regime. This is investigated in the next section.

\section{Application to LS 5039}

The influence of anisotropic scattering on the emission from gamma-ray binaries can be sketched from the results of the previous section. If the high-energy emission is due to inverse Compton scattering off electrons co-rotating with the binary, the viewing angle of the observer will vary with orbital phase, inducing changes in the observed spectrum - all other things being set equal (particle distribution and location, distance to the star, etc).

Anisotropic scattering will influence the emission from systems with high inclinations most, if the electrons are located in the orbital plane. At low inclinations, the changes are expected to be minor as the scattering angle $\psi$ stays close to $\pi / 2$. On the other hand, for high inclinations the inverse Compton spectrum may change significantly between inferior and superior conjunctions. The emission will be intense and soft at the time of maximum attenuation by pair production, and low and hard at the time of minimum attenuation. Anisotropic inverse Compton emission combined with attenuation of VHE photons can therefore play a significant part in (1) reducing the amplitude of the variations expected from a simple attenuation model and in (2) hardening the spectrum at high flux states compared to expectations from a calculation assuming an isotropic flux.

The LS 5039 system is an ideal testbed. The massive star has an O6.5V spectrum $\left(T_{\star}=39000 \mathrm{~K}, R_{\star}=9.3 R_{\odot}, M_{\star}=23 M_{\odot}\right)$ in a 3.9 day eccentric orbit $(e=0.35)$ with its compact companion (Casares et al. 2005). A diagram of the binary orbit oriented on the sky is shown in Fig. 2. The measured radial velocity of the $\mathrm{O}$ star constrains the inclination to about $60^{\circ}$ for a neutron star companion and about $20^{\circ}$ for a black hole. The compact star moves from one to three stellar radii from the surface of the massive star.

The intensity and spectral variations have been wellestablished in LS 5039 by HESS observations, concluding that pure attenuation of a constant VHE spectrum could not explain the observations to satisfaction (see Sect. 1). Given the above

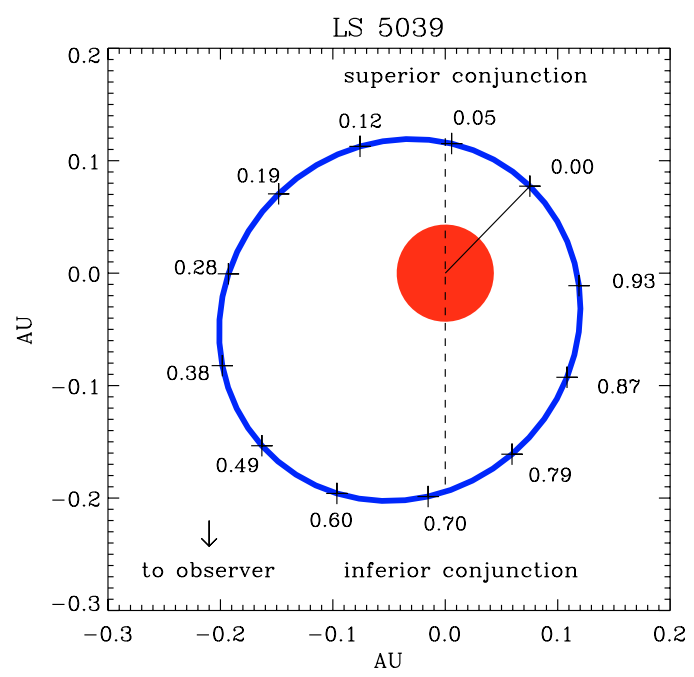

Fig. 2. The binary orbit of LS 5039 as seen from directly above. The O6.5V star radius is to scale. The binary orientation is set for an observer at the bottom of the diagram. The binary inclination on the plane of the sky is not taken into account. The numbers indicate the orbital phase (mean anomaly) at various positions. Periastron passage is indicated by a full line (orbital phase $\phi_{\text {orb }}=0$ ). The dashed line is the line of conjunctions ( $\phi_{\text {sup }} \approx 0.06, \phi_{\text {inf }} \approx 0.72$ ). The orbital parameters are taken from Casares et al. (2005).

discussion, this section examines whether taking anisotropic scattering into account improves agreement.

\subsection{The radiating electrons}

Two main assumptions are made to calculate the emission. First, the electrons are assumed to scatter radiation at the location of the compact object, in a small region compared to the orbital separation. This is a very good approximation in the pulsar wind nebula scenario where the highest energy electrons emit the gamma-ray radiation close to the shock. (The cooled electrons then emit in radio well away from the system.) This may or may not be appropriate in the case of a relativistic jet, where 
emission can occur at various distances along the outflow. This is discussed further Sect. 3.4.

Second, the adopted distribution of particles is the steadystate distribution for constant injection of particles, taking synchrotron and inverse Compton losses into account. The magnetic field in the radiating zone is assumed to be homogeneous. The radiative losses occur on very short timescales compared to the orbital timescale so the steady-state approximation is justified except for low-energy particles whose radiative timescale becomes longer than their escape timescale from the radiating zone. This occurs at $\gamma_{\mathrm{e}} \approx 10^{3}$ (see below). The injection spectrum is a power law $\mathrm{d} n_{\mathrm{e}} \propto \gamma_{\mathrm{e}}^{-p} \mathrm{~d} \gamma_{\mathrm{e}}$ with an exponential cutoff at the maximum $\gamma_{\max }$ allowed by comparing acceleration and radiative timescales.

The minimum acceleration timescale for $\mathrm{TeV}$ electrons $\left(\gamma_{6}=\right.$ $\left.10^{6}\right)$ is set by the gyrofrequency and is $t_{\mathrm{acc}} \approx 0.06 \gamma_{6} / B_{1} \mathrm{~s}$ with $B_{1}=1 \mathrm{G}$ the magnetic field intensity. The synchrotron cooling timescale is $t_{\mathrm{S}} \approx 770 / B_{1}^{2} \gamma_{6} \mathrm{~s}$. For electrons with Lorentz factors $\gamma_{\mathrm{e}}>\gamma_{\mathrm{KN}} \approx 6 \times 10^{4} T_{\star, 4}^{-1}$, inverse Compton scattering of stellar photons occurs in the Klein-Nishina regime. The corresponding timescale is $t_{\mathrm{IC}} \approx 20 \gamma_{6} d_{0.1}^{2} /\left[\ln \gamma_{6}+1.4\right]\left(T_{\star, 4} R_{\star, 10}\right)^{2} \mathrm{~s}$ (Blumenthal \& Gould 1970) with $T_{\star, 4}=40000 \mathrm{~K}, R_{\star, 10}=$ $10 R_{\odot}$, and $d_{0.1}$ is the orbital separation in units of $0.1 \mathrm{AU}$ (the LS 5039 orbital separation at periastron).

The steady-state distribution derives from a comparison of these three timescales. Synchrotron losses dominate the inverse Compton losses above a critical $\gamma_{\mathrm{S}}$ given by $\left(t_{\mathrm{S}}=t_{\mathrm{IC}}\right)$ :

$\gamma_{\mathrm{S}} \approx 6 \times 10^{6}\left(T_{\star, 4} R_{\star, 10}\right) /\left(B_{1} d_{0.1}\right)$.

At the highest energies, $\gamma_{\max }$ is therefore set by synchrotron losses $\left(t_{\mathrm{acc}}=t_{\mathrm{S}}\right)$, which gives $\gamma_{\max } \approx 1.2 \times 10^{8} B_{1}^{-1 / 2}$. Assuming continuous injection of electrons with a $\gamma_{\mathrm{e}}^{-p}$ spectrum, the steady-state distribution is steepened by synchrotron losses between $\gamma_{\mathrm{S}}$ and $\gamma_{\max }$ to a $\gamma_{\mathrm{e}}^{-p-1}$ power-law. Inefficient KleinNishina losses dominate between $\gamma_{\mathrm{KN}}$ and $\gamma_{\mathrm{S}}$, producing a hard spectrum mirroring the decrease in the energy loss rate with increasing $\gamma_{\mathrm{e}}$ in the Klein-Nishina regime. Below $\gamma_{\mathrm{KN}}$, inverse Compton losses in the Thomson regime result in a $\gamma_{\mathrm{e}}^{-p-1}$ powerlaw as in the synchrotron case.

Steady-state distributions obtained using a full numerical calculation follow the main characteristics outlined above very well (Fig. 3, see also Moderski et al. 2005). The inverse Compton losses are treated in the isotropic approximation since the magnetic field will quickly randomize particle directions. The particles see, on average, the equivalent of an isotropic radiation field; but the inverse Compton spectrum received by an observer at a fixed location changes with viewing angle. In Fig. 3, the injection is a power law $\gamma_{\mathrm{e}}^{-p}$ with $p=2$ and the distribution between $\gamma_{\mathrm{KN}}$ and $\gamma_{\mathrm{S}}$ is roughly proportional to $\gamma_{\mathrm{e}}^{-1.3}$. The slope of this distribution depends on the slope of the injected spectrum. For power-law injections $\gamma_{\mathrm{e}}^{-p}$ with hard indices $(p<2)$, the slope between $\gamma_{\mathrm{KN}}$ and $\gamma_{\mathrm{S}}$ tends to $\gamma_{\mathrm{e}}^{-1}$. For soft indices $p>2$, the hardening gradually disappears, reaching $\gamma_{\mathrm{e}}^{-2}$ between $\gamma_{\mathrm{KN}}$ and $\gamma_{\mathrm{S}}$ for an injection with $p=3$. As discussed below, the observations of LS 5039 constrain $p$ to about 2.

This steady-state distribution is a very good approximation of the more detailed pulsar wind model of Dubus (2006b) for electrons with $\gamma_{\mathrm{e}} \gtrsim 10^{3}$ : lower energy electrons escape from the vicinity of the pulsar without radiating much of their energy. More generally, this distribution should apply equally well to any leptonic model assuming a constant injection of non-thermal particles cooling in the vicinity of the compact object via synchrotron and inverse Compton radiation.

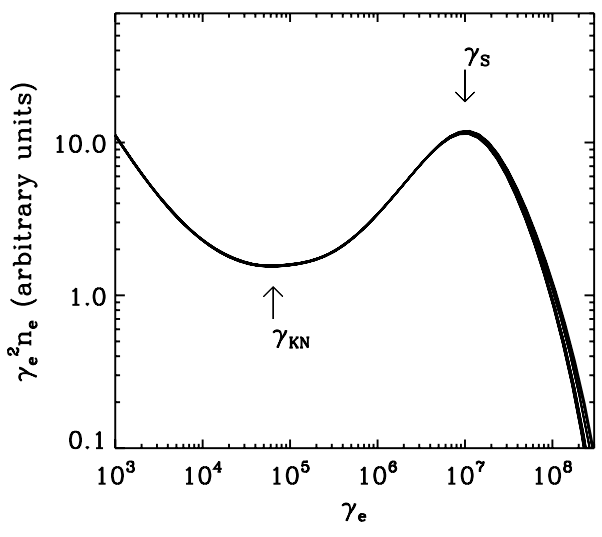

Fig. 3. Steady-state electron distribution $N_{\mathrm{e}}$ along the orbit of LS 5039. The injection spectrum is a $\gamma_{\mathrm{e}}^{-2}$ power law with an exponential cutoff at $\gamma_{\mathrm{e}} \approx 10^{8}$ (see Sect. 3.1). The magnetic field varies as $B=0.8 d_{0.1}^{-1} \mathrm{G}$, where $d_{0.1}$ is the orbital separation in units of $0.1 \mathrm{AU}$. Inverse Compton losses in the Thomson regime $\left(\gamma_{\mathrm{e}}<\gamma_{\mathrm{KN}}\right)$ and synchrotron losses $\left(\gamma_{\mathrm{e}}>\right.$ $\gamma_{\mathrm{S}}$ ) steepen the index of the injected distribution by one to $N_{\mathrm{e}} \propto \gamma_{\mathrm{e}}^{-3}$. Inverse Compton losses in the Klein-Nishina regime dominate between $\gamma_{\mathrm{KN}}<\gamma_{\mathrm{e}}<\gamma_{\mathrm{S}}$, causing a hardening of the distribution (Moderski et al. 2005). The steady-state distribution varies little with orbital phase since $\gamma_{\mathrm{S}} \propto(B d)^{-1}$ stays constant: the changes with orbital phase produce only a slight thickening of the line in the figure above.

\subsection{Compact pulsar wind nebula: orbital light curve}

With the inverse Compton losses fixed by the geometry, the only remaining free parameters are the slope of the injected power law, the total energy in radiating electrons, and the value of the magnetic field. In the case of a compact pulsar wind nebula, the magnetic field is determined by the conditions at the pulsar-wind termination shock. Its intensity sets $\gamma_{\mathrm{S}}$, which in turn will fix the frequency above which a break will be seen in the VHE gammaray spectrum. In principle, $B$ may vary with orbital phase as the eccentric orbit brings the pulsar at various radii in the stellar wind. However, the magnetic field intensity is inversely proportional to the shock distance from the pulsar, and the latter is roughly proportional to the orbital separation so that $B \propto 1 / d$ (see e.g. Dubus 2006b). In this case, the distribution of particles will not change along the orbit as $\gamma_{\mathrm{S}} \propto(B d)^{-1}$.

Figure 4 shows the expected light curve at different orbital phases with $B=0.8 \mathrm{G}$ at periastron and $p=2$ (using the electron distribution shown in Fig. 3). The orbital elements were computed as in Dubus (2006a). The unabsorbed intensity is high close to superior conjuction and small at inferior conjunction, as explained in Sect. 2.3. The angle to the observer varies between $30^{\circ}$ and $150^{\circ}$, whereas the angular size of the star at the compact object is $30^{\circ}$ at most: the finite size of the star, taken into account in the calculation, has a minor effect on the results. The attenuation light curve, computed following Dubus (2006a) is also shown. It peaks at the inferior conjunction where attenuation is minimum.

The light curve including both anisotropic emission and attenuation by pair production reproduces the observed light curve well. Most notably, the combination of low attenuation, increasing photon density, and a hard inverse Compton spectrum produces a small peak after inferior conjunction that appears to be present in the HESS observations. The peak is a key feature of this model. This light curve is very robust against changes in the value of the magnetic field used, or even in the type of particle distribution used. At higher inclinations, a weaker peak appears before inferior conjunction as the variations in viewing angle 

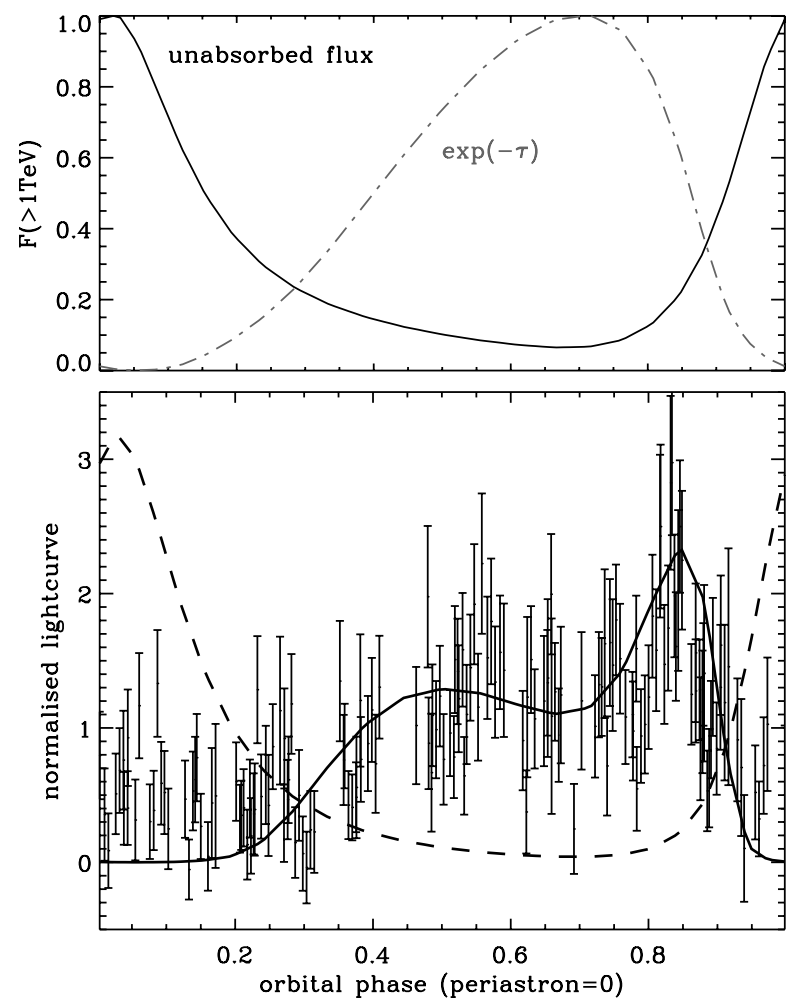

Fig. 4. Predicted orbital light curves for LS 5039 in the case of a neutron star $\left(i=60^{\circ}\right)$. Top panel: the integrated photon rate above $1 \mathrm{TeV}$ (full line) due to anisotropic inverse Compton scattering and the transmission $\exp \left(-\tau_{\gamma \gamma}\right)$ for the pair production process, also integrated above $1 \mathrm{TeV}$. Inverse Compton scattering is minimum at inferior conjunction $\left(\phi_{\text {orb }} \approx 0.72\right.$, see Fig. 2$)$. The absorption due to pair production is also minimum at this time. Bottom panel: the resulting orbital light curve (full line) compared to the HESS observations. Combining anisotropic inverse Compton emission and attenuation by pair production produces a peak at $\phi_{\text {orb }} \approx 0.8$ consistent with the observations. The agreement is good except at periastron where cascade emission (ignored here) may be important. The dashed line shows the photon rate in GLAST above $1 \mathrm{GeV}\left(\mathrm{ph} \mathrm{cm}^{-2} \mathrm{~s}^{-1}\right)$. The model predicts a peak in the GLAST light curve close to periastron and a minimum at inferior conjunction. The normalizations are arbitrary. The light curves are calculated using the electron distributions shown in Fig. 3.

cause a larger drop in inverse Compton emission at $\phi_{\text {orb }}=0.72$. However, this model still predicts little to no flux at and after periastron because of the very strong attenuation of the emission emitted around the pulsar. A possible explanation is that a pair cascade develops.

The light curve above $1 \mathrm{GeV}$ is also plotted in Fig. 4. Attenuation is negligible and the variations mostly follow the photon density, with some modifications due to the anisotropy; for instance, the minimum is at inferior conjuction. GLAST should therefore see a modulation in the flux from LS 5039 with a peak close to periastron and a minimum at inferior conjunction, almost anti-correlated with the HESS modulation.

A similar light curve has been obtained by Bednarek (2007), using a complex Monte-Carlo code simulating the effects of anisotropic scattering and the development of cascades. However, it wrongly interprets the GLAST modulation as due to stronger cascade emission close to periastron. As described above, the modulation is due to a combination of increased seed photon density and anisotropic effects and not to cascade emission $^{1}$.

\subsection{Compact pulsar wind nebula: phase-resolved spectra}

Figure 5 shows the evolution of the attenuated and unattenuated spectra with orbital phase. These were used to produce the light curves shown in Fig. 4. The spectra display a complex interplay between the varying threshold for pair production, the high absorption it causes at superior conjunction when the inverse Compton flux is high, and the weaker but harder inverse Compton emission at inferior conjunction. The variations in the $\mathrm{GeV}$ (GLAST) range have a very large amplitude, with a flat spectrum at the highest intensities and a hard spectrum at low intensities. This should easily be accessible to GLAST in the very near future (Dubois 2006). Note that synchrotron emission contributes significantly to the emission below one $\mathrm{GeV}$ and that this is not taken into account in this light curve. Its impact is to soften the spectrum and reduce the amplitude of the variations below a GeV (see Sect. 3.4 below and Fig. 6).

The attenuated spectrum averaged over the full orbit is shown in Fig. 6. The hitherto puzzling drop between the EGRET and HESS spectra is reproduced well by the model without invoking a cascade. The inverse Compton spectrum by itself underestimates the EGRET flux but, taking the synchrotron emission from the electrons into account using the adopted magnetic field intensity ( $B=0.8 \mathrm{G}$ at periastron and varying as $1 / d$ ), the calculated synchrotron emission produces a very good match to both the EGRET and HESS spectra. Note that the average HESS spectrum is not shown for reasons of clarity in Fig. 6 but is close to the "high" state spectrum (see below), with a slightly higher luminosity.

The two average spectra for the phase intervals of the HESS "high" $\left(0.45<\phi_{\text {orb }}<0.9\right)$ and "low" states $\left(\phi_{\text {orb }}<0.45\right.$ or $\phi_{\text {orb }}>0.9$ ) spectra are also shown in Fig. 6 (Aharonian et al. 2006). Reproducing the cutoff in the high-state HESS spectrum strongly constrains the magnetic field intensity to $\approx 0.8 \mathrm{G}$ at periastron. A higher magnetic field moves the cutoff to lower energies and is inconsistent with the data. A lower $B$ moves the cutoff to higher energies and hardens the spectrum too much. The highstate spectrum is sensitive to the value of $B$, with the acceptable range only $B=0.8 \pm 0.2 d_{0.1}^{-1} \mathrm{G}$. Outside of this range the fit does not go through the error bars of the HESS data points.

The synchrotron emission contributes significantly below $1 \mathrm{GeV}$, diluting the hardening of the spectrum around $\phi=0.7$ expected from pure inverse Compton emission. Actually, a softening is predicted below a few GeV. The GLAST light curve shown in Fig. 4 is not noticeably changed (on a linear scale) by taking synchrotron emission into account. The hard electron distribution, naturally resulting from inefficient Klein-Nishina losses here, is instrumental in obtaining the flat spectrum in the HESS range. The range $\gamma_{\mathrm{KN}}<\gamma_{\mathrm{e}}<\gamma_{\mathrm{S}}$ of this hard distribution depends upon the value of the magnetic field, but its shape is independently set by the index $p$ of the injected power law $\gamma_{\mathrm{e}}^{-p}$. With $p \lesssim 1.7$, the predicted HESS spectrum is too hard and the emission in the EGRET band is too low. With $p \gtrsim 2.3$, the

\footnotetext{
${ }^{1}$ Bednarek (2007) also confused the phases of inferior and superior conjunctions (Fig. 2). The compact object is on the near side of the orbit (inferior conjunction) at phases $0.4-0.8$ so that the broad maximum is not due to the stronger Compton scattering expected when the object is behind the star (see Fig. 5). Similarly, the dip at phase 0.7 is caused by stronger absorption (expected at superior conjunction): it actually occurs at the phase of minimum absorption and is due to the lower Compton emissivity at inferior conjunction, as described above.
} 

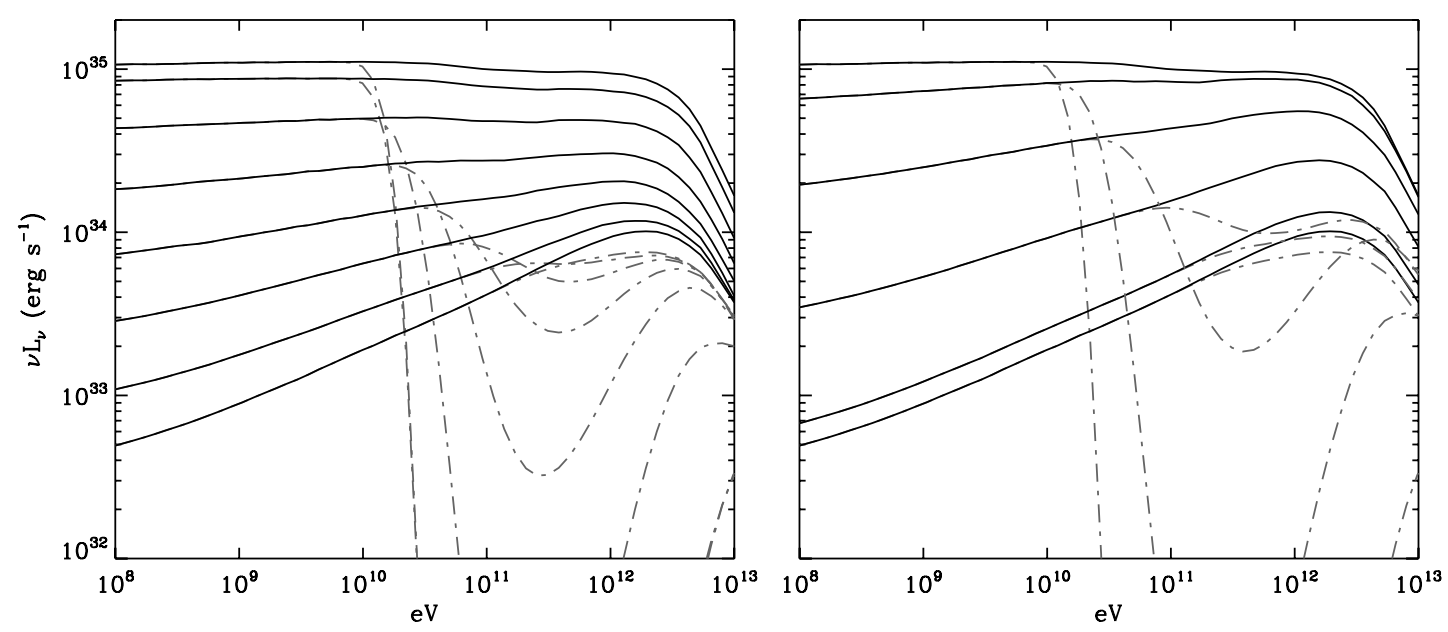

Fig. 5. Evolution of the model inverse Compton spectrum with orbital phase in LS 5039 (neutron star case). The intrinsic emission spectra are shown with full lines and the dashed lines show the spectra after attenuation by pair production on stellar photons during the propagation of the gamma rays through the system. The underlying electron distributions are those shown in Fig. 3. Left panel: from top to bottom the spectra correspond to orbital phases $\phi_{\mathrm{orb}}=0.03,0.09,0.15,0.24,0.34,0.44,0.56$ and 0.66 (see Fig. 2). Right panel: the plotted orbital phases from bottom to top are $0.66,0.76,0.85,0.91,0.97$ and 0.03 .

predicted HESS spectrum is too soft and the EGRET emission is too large. Therefore, the slope of the injected power law is constrained to $p=2 \pm 0.3$.

Besides the magnetic field intensity and slope of injected electrons, the other free parameter is the normalization of the electron distribution. The fit was obtained for a total energy in electrons from $\gamma_{\mathrm{e}}=10^{3}$ to $+\infty$ of $3 \times 10^{37} \mathrm{erg}$. This energy corresponds to the injection of $10^{36} \mathrm{erg} \mathrm{s}^{-1}$ in particles, assuming an escape timescale from the radiative zone of $30 \mathrm{~s}$ (longer than the radiative timescale under consideration). In the pulsar wind nebula, the shocked electrons have a bulk velocity $\approx \mathrm{c} / 3$ so that the escape timescale corresponds to a radiating zone of $3 \times 10^{11} \mathrm{~cm}$, comparable to the shock size found for typical wind parameters in LS 5039 (Dubus 2006b). The estimated injection energy rate is consistent with a reasonable pulsar spindown power, such as measured in PSR B1259-63 (Manchester et al. 1995).

The low-state spectrum is responsible for most of the orbitaveraged emission in the EGRET range, which is nicely fit by the model. However, the HESS low-state spectrum is not satisfactory. This spectrum corresponds to phases where the intrinsic inverse-Compton emission is both soft, like the observed spectrum, and intense. The intrinsic emission is actually strongest at the times of highest attenuation so that the two effects compensate somewhat. However, the cross-section for pair production drops above a few TeV. Therefore, the predicted phase-averaged low state is not a pure power law but still shows hints of an attenuation line with a kink at high energies. Changes in the electron distribution may also help to reduce the discrepancy. A cutoff at a lower $\gamma_{\mathrm{e}}$ (i.e. a higher magnetic field) than that shown in Fig. 3 would yield better agreement if it occurred at the appropriate orbital phases. However, at this stage it appears more reasonable to investigate first the impact of pair cascading on this spectrum, as this is required to explain the detection at periastron.

The model contains only three parameters: the slope of the injected power-law, the particle distribution normalization, and the magnetic field intensity at periastron (or any other arbitrary orbital phase). The shape of the particle distribution and the associated emission along the orbit are then unequivocally predicted. The parameters were adjusted so as to fit the high-state HESS

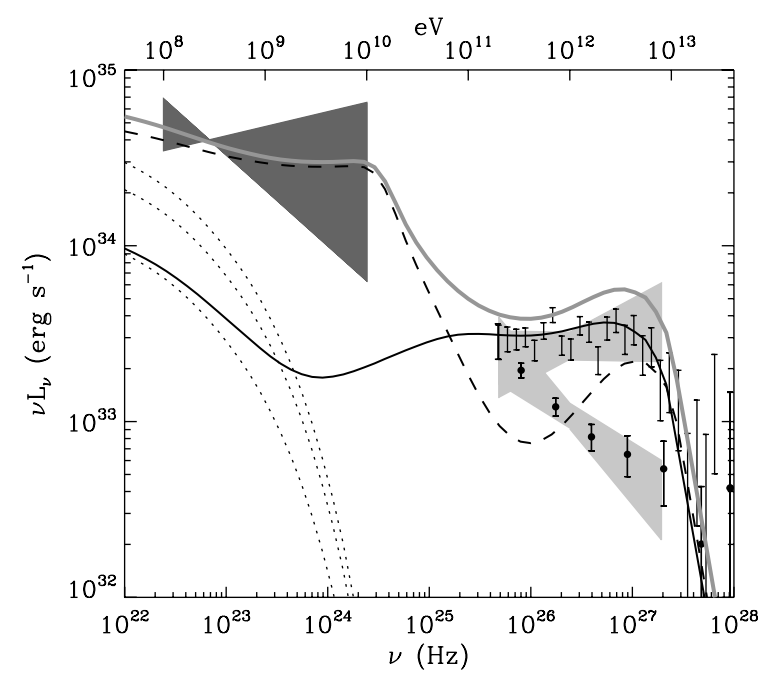

Fig. 6. Comparison with the EGRET and HESS observations of the LS 5039 model spectra for a neutron star. The EGRET bowtie is in dark grey and the HESS high-state and low-state bowties are in light grey (Hartman et al. 1999; Aharonian et al. 2006). The corresponding HESS deconvolved spectral points are also shown (with a dot identifying the low-state points). Fluxes have been transformed to luminosities assuming a distance of $2.5 \mathrm{kpc}$ (Casares et al. 2005). The full grey line is the average spectrum calculated using the results of Fig. 5. It reproduces the drop in flux from EGRET to HESS well (the average HESS spectrum is close to the high-state spectrum shown). The highstate spectrum (full dark line) is reproduced well provided the magnetic field at periastron is lower than $0.8 \mathrm{G}$. The low-state spectrum (dashed dark line) is not reproduced well, possibly because cascade emission contributes significantly at these orbital phases where pair production is very important or because the electron distribution varies along the orbit. Here, the synchrotron emission from the electrons is taken into account with $B=0.8 d_{0.1}^{-1} \mathrm{G}$ as derived from the VHE spectrum. Its contribution to the spectra is shown by the dotted lines (from bottom to top: low-state, high-state, and orbital average).

spectrum. That this choice also fits the EGRET observations well gives strong support to this simple model, even if the low-state HESS spectrum is not reproduced to satisfaction. 


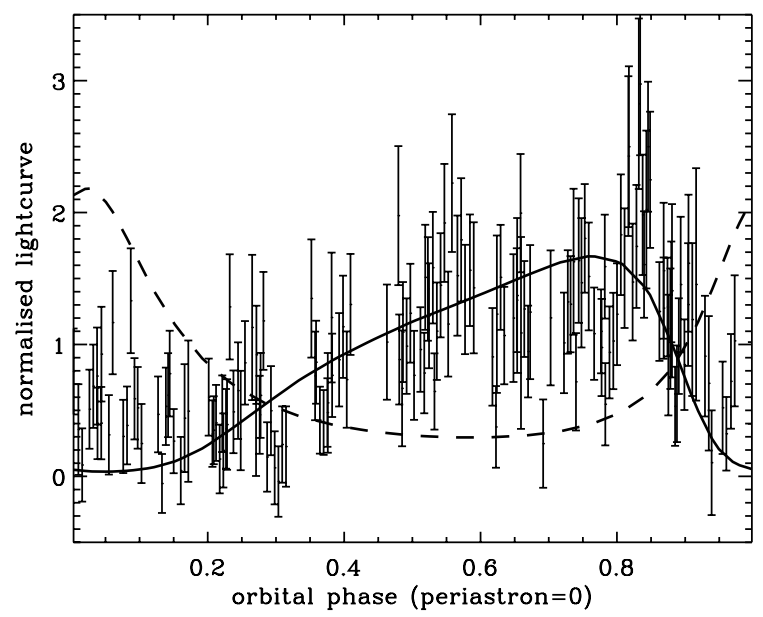

Fig. 7. Predicted orbital light curves for LS 5039 in the case of a black hole $\left(i=20^{\circ}\right)$. The full line is the integrated photon flux above $1 \mathrm{TeV}$ (HESS), and the dashed line is integrated above $1 \mathrm{GeV}$ (GLAST). The variations in viewing angle are reduced compared to the high inclination (neutron star) case (Fig. 4), and there is only one broad maximum in the HESS light curve. The electron distribution is calculated as described in Fig. 3 but using a constant magnetic field intensity of $0.8 \mathrm{G}$.

\subsection{Black hole jet?}

This section examines how the results are changed if the compact object is a black hole. The main effect is that consistency with radial velocity curves require the inclination to change to $20^{\circ}\left(4.5 M_{\odot}\right.$ black hole $)$. The variation in viewing angle is then reduced to the interval $70^{\circ}-110^{\circ}$. The electrons are still assumed to be accelerated in the vicinity of the black hole and to reach a steady-state distribution, such as the one described above. Here, the magnetic field has a fixed value as there is no a priori reason for it to change with the orbital separation. This gives a moderate change of a factor 2 in the break $\gamma_{S}$ of the electron distribution, because the orbital separation varies by a factor 2 , in contrast to the situation described in Fig. 3.

The orbital light curve and the spectra obtained with $B=$ $0.8 \mathrm{G}$ and $p=2$ are shown in Figs. 7-8. In contrast to the neutron star case, there is only one broad peak in the predicted HESS light curve. This is because the reduced variation of the viewing angle does not lead to a large drop in scattered flux at inferior conjunction. The small peak predicted at high inclinations (neutron star) can therefore be used as a discriminant between the two cases. The averaged spectra are much harder than in the neutron-star case. The amplitude of the variation at $\mathrm{GeV}$ energies is less than for a neutron star and the average flux overestimates the EGRET emission. The poor fit of the low-state spectrum remains. Both the light curve and spectra do not fit as well as those obtained in the neutron star case, but not so much as to exclude that LS 5039 is seen at a low inclination (and hence contains a black hole).

Emission from a relativistic jet may differ from the estimate above. Any Doppler boosting will change the observed spectrum. However, the resolved radio emission, if interpreted as a compact jet, only implies a moderate velocity and little boosting (Paredes et al. 2000). Modest Doppler (de)boosting may also be expected from the pulsar wind emission as its post-shock speed is approximately $c / 3$. More importantly, emission may occur all along the jet and not just be localized near the black hole. Far from the compact object, the viewing angle tends to become the

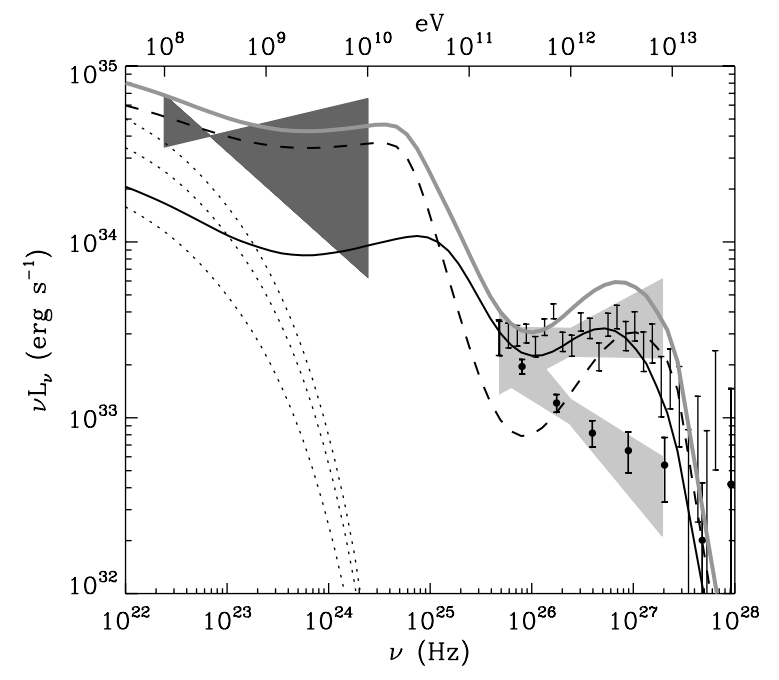

Fig. 8. Comparison with the EGRET and HESS observations of the LS 5039 model spectra for a black hole. The radiating electrons are injected in the immediate vicinity of the black hole. The magnetic-field intensity used to fit the high-state spectrum is $0.8 \mathrm{G}$, constant throughout the orbit. The injected electrons have a power law of index $p=2$. The line coding is the same as in Fig. 6.

inclination angle $(\psi \rightarrow i)$ regardless of orbital phase ${ }^{2}$. Hence, emission at progressively higher altitudes in the jet is influenced less and less by anisotropic effects. The emission is also less attenuated by pair production, with $\tau_{\gamma \gamma}$ negligible at heights $\gtrsim 1$ AU. If most of the emission occurs far in the jet, and assuming the electron distribution stays constant, the flux modulation is only linked to the stellar photon density. The result is a constant spectral shape, peak flux at periastron, and a trough at apastron. These are inconsistent with the observations. Therefore, a jet model for LS 5039 probably requires either (1) that most of the emission occurs close to the compact object in order to reproduce the orbital gamma-ray modulation via anisotropic scattering and attenuation or (2) that the emission occurs away in the jet and that some unspecified intrinsic mechanism changes the particle distribution and/or the radiation process.

\section{Conclusion}

The anisotropic behaviour of inverse Compton scattering has a major influence on the emission from gamma-ray binaries. In these sources, the massive star provides a large source of seed photons with energies around an electron volt. If high-energy electrons are accelerated in the vicinity of the compact object, then the angle between the star, compact object, and observer changes with orbital phase. The variation in viewing angle leads to a strong modulation in both the intensity and spectral shape of the scattered radiation.

Scattering stellar photons to the $\mathrm{TeV}$ range requires very energetic electrons with Lorentz factors $\gamma_{\mathrm{e}} \approx 10^{6}-10^{7}$. The scattering therefore occurs in the Klein-Nishina regime. In this case, the anisotropy results, at inferior conjunction, in a harder and fainter spectrum than predicted using an isotropic approximation

2 Note that two errors have slipped by in Dubus (2006a) when dealing with the case of a VHE source perpendicular to the orbital plane. In the last equation of A.2 the angle for emission perpendicular to the plane is given as $\cos \psi=\left(d_{0} / d\right) \cos \psi_{0}=\left(d_{0} / d\right) \sin \theta \sin i$ but should be $\cos \psi=$ $\left(d_{0} / d\right) \sin \theta \sin i-(z / d) \cos i$. The other is that Fig. 8 (attenuation with height) was calculated at a fixed viewing angle of $76^{\circ}$. The conclusions are unchanged. 
for the incoming photons. Crucially, inferior conjunction also corresponds to the phase at which the produced VHE gammarays are less attenuated by pair production on stellar photons. At other phases the emitted spectrum is close to the one obtained using the isotropic photon field approximation and can be severely attenuated by pair production. The result is a complex interplay that reduces the amplitude of the variations expected from a pure attenuation model and a hardening at inferior conjunction.

The LS 5039 light curve and spectra were modelled using a simple leptonic model. The electrons are assumed to be accelerated efficiently in a small zone in the vicinity of the compact object with a standard $\gamma_{\mathrm{e}}^{-p}$ power law. Radiative losses due to inverse Compton emission and synchrotron emission generate a distinctive steady-state electron distribution in this environment dominated by stellar photons. The distribution has a prominent hardening between the energy at which inverse Compton losses enter the Klein-Nishina regime $\left(\gamma_{\mathrm{KN}} \approx 6 \times 10^{4}\right.$ in LS 5039) and the energy at which synchrotron losses take over $\left(\gamma_{S} \approx 10^{7}\right.$ for a $1 \mathrm{G}$ field). This is for instance the distribution found in the vicinity of the pulsar wind shock but it applies equally well to any leptonic model where particles are accelerated close to the compact object. The magnetic field was allowed to vary as the inverse of the orbital separation, as expected from a pulsar wind nebula. The model has only three parameters: the intensity of the magnetic field, the normalization of the electron distribution and the slope $p$ of the injected power-law $\gamma_{\mathrm{e}}^{-p}$.

The cutoff in the very high-energy gamma-ray spectrum is very sensitive to the magnetic-field intensity, via the location of $\gamma_{\mathrm{S}}$ in the electron distribution. Fitting the high-state spectrum seen by HESS gives a rather constrained magnetic-field intensity at periastron of $0.8 \pm 0.2 \mathrm{G}$. This value compares well with the values found using simple pulsar wind models that give 5 $\left(\dot{E}_{36} \sigma_{3}\right)^{1 / 2} R_{11}^{-1} \mathrm{G}$, where $\dot{E}_{36}$ is the pulsar spindown power in units of $10^{36} \mathrm{erg} \mathrm{s}^{1}, \sigma_{3}$ is the ratio of magnetic to kinetic energy in the pulsar wind in units of $10^{-3}$, and $R_{11}$ is the distance of the shock to the pulsar in units of $10^{11} \mathrm{~cm}$. Fitting the HESS high-state spectrum also sets the injection slope to $p=2 \pm 0.3$, close to the canonical value for shock acceleration. The normalization of the electron distribution implies an injection rate of $10^{36} \mathrm{erg} \mathrm{s}^{-1}$ for a radiative zone of $310^{11} \mathrm{~cm}$. These results are remarkably consistent with the expectations for a pulsar wind model.

The spectrum is also found to fit the EGRET observations well, adding credence to this simple approach. The model predicts a strong variation in the GLAST band with a softening from high to low flux below one $\mathrm{GeV}$ (where synchrotron emission dominates the spectrum) but a hardening above a $\mathrm{GeV}$ (where inverse Compton emission dominates the spectrum). The HESS low-state spectrum has not been explained to satisfaction. The model fits the EGRET measurements but produces too many gamma rays at 5-10 TeV. A possible solution is a more complex orbital phase-dependence of the electron distribution at selected phases. Another solution is that the low-state spectrum corresponds to phases of strong attenuation and that emission from the created pairs contribute significantly to the spectrum. Additional HESS observations near minimum flux would be welcomed.

The orbital modulation of the HESS emission is reproduced. A well-defined peak is predicted between phases $0.7-0.9$ for which evidence may already be seen in the data. The light curve at GLAST energies is anti-correlated with the HESS light curve and has a peak at periastron, where the stellar photon density is maximum, and a minimum at inferior conjunction because of the anisotropic effects in inverse Compton scattering. The GLAST spectrum below $1 \mathrm{GeV}$ should be influenced by the tail of the synchrotron emission from the highest-energy electrons. The peak synchrotron emission is at about $100 \mathrm{MeV}$ for maximally accelerated electrons, regardless of magnetic field. Hence, if this component is detected, it will provide evidence that electrons are indeed accelerated with extreme efficiency in this source.

Similar results for the magnetic field intensity and particle energy are found when a lower inclination is used, i.e. implying a black hole compact object rather than a neutron star. In this case, the emission is thought to arise from a relativistic jet powered by accretion onto the black hole. Within the assumptions of this work on the particle distribution, it is difficult to argue that a significant part of the emission occurs far along a jet since this does not naturally reproduce either the spectrum or the light curve measured by HESS. Most of the emission should still occur close to the compact object. However, unlike in the case of a pulsar wind nebula, there are no independent theoretical expectations in support of the magnetic-field intensity (certainly lower than its equipartition value in the accretion flow) and particle energy that are derived. Therefore, the pulsar wind nebula model appears to be favoured.

Despite the complexity of the phenomena involved in pulsar wind nebula emission, it is found that the peculiar environment of a gamma-ray binary, most prominently the enormous luminosity of the massive companion, severely constrains the number of degrees-of-freedom in the model. A simple model suffices to reproduce most of the observations. The value of the magnetic field at the shock is found to be tightly constrained by the HESS observations to $0.8 \pm 0.2 \mathrm{G}$ and the injection spectrum slope to $p=2 \pm 0.3$. These results confirm that gamma-ray binaries are promising sources for studying the environment of pulsars on very small scales.

Acknowledgements. GD acknowledges support from the Agence Nationale de la Recherche and comments by $\mathrm{B}$. Giebels on an early draft.

\section{Appendix A: Inverse Compton spectrum for a mono-energetic beam of photons}

The purpose of this Appendix is first to carry out the integration set out in Eq. (5) and second to give an expression valid in the Klein-Nishina regime for the total spectrum emitted by a single electron scattering a mono-energetic beam of photons (Eq. (7)). The fraction of scattered photons per time, energy, and steradian is given by Eq. (6), which can be expanded using Eqs. (1-5)

$$
\begin{aligned}
\frac{\mathrm{d} N}{\mathrm{~d} t \mathrm{~d} \epsilon_{1} \mathrm{~d} \Omega_{1}}= & \frac{r_{\mathrm{e}}^{2} c\left(1-\beta \mu_{0}\right)}{2 \gamma_{\mathrm{e}}\left(1-\beta \mu_{1}\right)} \iiint\left(\frac{\epsilon_{1}^{\prime}}{\epsilon^{\prime}}\right)^{2}\left(\frac{\epsilon_{1}^{\prime}}{\epsilon^{\prime}}+\frac{\epsilon^{\prime}}{\epsilon_{1}^{\prime}}-\sin ^{2} \Theta^{\prime}\right) \\
& \times \delta\left(\epsilon^{\prime}-\epsilon_{0}^{\prime}\right) \delta\left(\mu^{\prime}-\mu_{0}^{\prime}\right) \delta\left(\phi^{\prime}-\phi_{0}^{\prime}\right) \\
& \times \delta\left(\epsilon_{1}^{\prime}-\frac{\epsilon^{\prime}}{1+\frac{\epsilon^{\prime}}{m_{\mathrm{e}} c^{2}}\left(1-\mu_{\Theta^{\prime}}\right)}\right) \mathrm{d} \epsilon^{\prime} \mathrm{d} \mu^{\prime} \mathrm{d} \phi^{\prime}
\end{aligned}
$$

where primed (unprimed) quantities are measured in the electron (observer) frame, $\mu_{\Theta^{\prime}} \equiv \cos \Theta^{\prime}=\mu^{\prime} \mu_{1}^{\prime}+\sin \theta^{\prime} \sin \theta_{1}^{\prime} \cos \left(\phi_{1}^{\prime}-\phi^{\prime}\right.$ ), $\mu^{\prime}=\cos \theta^{\prime}, \mu_{0}=\cos \theta_{0}, \mu_{0}^{\prime}=\cos \theta_{0}^{\prime}$ etc. Re-arranging the last Dirac and performing the three integrations yields

$$
\begin{aligned}
\frac{\mathrm{d} N}{\mathrm{~d} t \mathrm{~d} \epsilon_{1} \mathrm{~d} \Omega_{1}}= & \frac{r_{\mathrm{e}}^{2} c\left(1-\beta \mu_{0}\right)}{2 \gamma_{\mathrm{e}}\left(1-\beta \mu_{1}\right)} \\
& \times\left[1+\mu_{\Theta_{0}^{\prime}}^{2}+\left(\frac{\epsilon_{1}^{\prime}}{m_{\mathrm{e}} c^{2}}\right)^{2} \frac{\left(1-\mu_{\Theta_{0}^{\prime}}\right)^{2}}{1-\frac{\epsilon_{1}^{\prime}}{m_{\mathrm{e}} c^{2}}\left(1-\mu_{\Theta_{0}^{\prime}}\right)}\right] \\
& \times \delta\left(\frac{\epsilon_{1}^{\prime}}{1-\frac{\epsilon_{1}^{\prime}}{m c^{2}}\left(1-\mu_{\Theta_{0}^{\prime}}\right)}-\epsilon_{0}^{\prime}\right) .
\end{aligned}
$$


The integration over $\Omega_{1}$ to obtain the full spectrum of radiation emitted by the electron is simplified if $\gamma_{\mathrm{e}} \gg 1$. In that case,

$$
\begin{aligned}
\mu_{\Theta_{0}^{\prime}}= & \mu_{0}^{\prime} \mu_{1}^{\prime}+\sin \theta_{0}^{\prime} \sin \theta_{1}^{\prime} \cos \left(\phi_{1}^{\prime}-\phi_{0}^{\prime}\right) \\
= & \frac{\mu_{0}-\beta}{1-\beta \mu_{0}} \frac{\mu_{1}-\beta}{1-\beta \mu_{1}} \\
& +\frac{1}{\gamma_{\mathrm{e}}^{2}} \frac{\sin \theta_{1}}{1-\beta \mu_{1}} \frac{\sin \theta_{0}}{1-\beta \mu_{0}} \cos \left(\phi_{1}-\phi_{0}\right) \approx \mu_{0}^{\prime} \mu_{1}^{\prime},
\end{aligned}
$$

which is equivalent to saying the outgoing photon is emitted along the direction of electron motion when $\gamma_{\mathrm{e}} \gg 1$. The last Dirac can then be rewritten as a function of $\mu_{1}$ :

$$
\begin{aligned}
\frac{\mathrm{d} N}{\mathrm{~d} t \mathrm{~d} \epsilon_{1} \mathrm{~d} \Omega_{1}}= & \frac{r_{\mathrm{e}}^{2} c\left(1-\beta \mu_{0}\right)}{2 \gamma_{\mathrm{e}}\left(1-\beta \mu_{1}\right)} \\
& \times\left[1+\mu_{\Theta_{0}^{\prime}}^{2}+\left(\frac{\epsilon_{1}^{\prime}}{m_{\mathrm{e}} c^{2}}\right)^{2} \frac{\left(1-\mu_{\Theta_{0}^{\prime}}\right)^{2}}{1-\frac{\epsilon_{1}^{\prime}}{m_{\mathrm{e}} c^{2}}\left(1-\mu_{\Theta_{0}^{\prime}}\right)}\right] \\
& \times \frac{\left[1-\frac{\gamma_{\mathrm{e}} \epsilon_{1}}{m_{\mathrm{e}} c^{2}}\left(1+\beta \mu_{0}^{\prime}-\left(\beta+\mu_{0}^{\prime}\right) \mu_{1}\right)\right]^{2}}{\left|\beta \gamma_{\mathrm{e}} \epsilon_{1}+\frac{\epsilon_{1}^{2}}{m_{\mathrm{e}} c^{2}} \mu_{0}^{\prime}\right|} \delta\left(\mu_{1}-x\right)(\mathrm{A} .4)
\end{aligned}
$$

with $x=\frac{1-\frac{\epsilon_{0}^{\prime}}{\gamma_{\mathrm{e}} \epsilon_{1}}+\frac{\epsilon_{0}^{\prime}}{m_{\mathrm{e}} c^{2}}\left(1+\beta \mu_{0}^{\prime}\right)}{\beta+\frac{\epsilon_{0}^{\prime}}{m_{\mathrm{e}} c^{2}}\left(\beta+\mu_{0}^{\prime}\right)}$.

The integration over $\Omega_{1}$ is now straightforward, giving for the total spectrum:

$$
\begin{aligned}
\frac{\mathrm{d} N}{\mathrm{~d} t \mathrm{~d} \epsilon_{1}}= & \frac{\pi r_{\mathrm{e}}^{2} c\left(1-\beta \mu_{0}\right)}{\gamma_{\mathrm{e}}(1-\beta x)} \\
& \times\left[1+\left(\frac{x-\beta}{1-x \beta}\right)^{2} \mu_{0}^{\prime 2}+\left(\frac{\gamma_{\mathrm{e}} \epsilon_{1}}{m_{\mathrm{e}} c^{2}}\right)^{2}\right. \\
& \left.\times \frac{\left[1+\beta \mu_{0}^{\prime}-\left(\beta+\mu_{0}^{\prime}\right) x\right]^{2}}{1-\frac{\gamma_{\mathrm{e}} \epsilon_{1}}{m_{\mathrm{e}} c^{2}}\left[1+\beta \mu_{0}^{\prime}-\left(\beta+\mu_{0}^{\prime}\right) x\right]}\right] \\
& \times \frac{\left[1-\frac{\gamma_{\mathrm{e}} \epsilon_{1}}{m_{\mathrm{e}} c^{2}}\left(1+\beta \mu_{0}^{\prime}-\left(\beta+\mu_{0}^{\prime}\right) x\right)\right]^{2}}{\left|\beta \gamma_{\mathrm{e}} \epsilon_{1}+\frac{\epsilon_{1}^{2}}{m_{\mathrm{e}} c^{2}} \mu_{0}^{\prime}\right|} .
\end{aligned}
$$

Relativistic kinematics gives the domain of variation of the scattered photon energy $\epsilon_{1}$ in the observer frame. The maximum $\epsilon_{+}$ and minimum $\epsilon_{-}$energies in the spectrum are

$$
\epsilon_{ \pm}=\frac{\left(1-\beta \mu_{0}\right) \epsilon_{0}}{1+\frac{\epsilon_{0}}{\gamma_{\mathrm{e}} m_{\mathrm{e}} c^{2}} \pm\left[\beta^{2}+2 \beta \mu_{0}\left(\frac{\epsilon_{0}}{\gamma_{\mathrm{e}} m_{\mathrm{e}} c^{2}}\right)+\left(\frac{\epsilon_{0}}{\gamma_{\mathrm{e}} m_{\mathrm{e}} c^{2}}\right)^{2}\right]^{1 / 2}} .
$$

The angle dependence of the maximum energy in the Thomson regime is $\left(1-\beta \mu_{0}\right)$. For high electron energies, in the KleinNishina regime, the maximum photon energy is limited to $\gamma_{\mathrm{e}} m_{\mathrm{e}} c^{2}$ and becomes almost independent of the angle.

\section{References}

Aharonian, F., Akhperjanian, A. G., Aye, K.-M., et al. 2005a, Science, 309, 746 Aharonian, F., Akhperjanian, A. G., Aye, K.-M., et al. 2005b, A\&A, 442, 1

Aharonian, F., Akhperjanian, A. G., Bazer-Bachi, A. R., et al. 2006, A\&A, 460, 743

Albert, J., Aliu, E., Anderhub, H., et al. 2006, Science, 312, 1771

Albert, J., Aliu, E., Anderhub, H., et al. 2007, ArXiv e-prints, 706

Ball, L., \& Kirk, J. G. 2000, Astropart. Phys., 12, 335

Bednarek, W. 2007, A\&A, 464, 259

Blumenthal, G. R., \& Gould, R. J. 1970, Rev. Mod. Phys., 42, 237

Bogovalov, S. V., \& Aharonian, F. A. 2000, MNRAS, 313, 504

Böttcher, M., \& Dermer, C. D. 2005, ApJ, 634, L81

Casares, J., Ribó, M., Ribas, I., et al. 2005, MNRAS, 364, 899

Dermer, C. D., \& Böttcher, M. 2006, ApJ, 643, 1081

Dhawan, V., Mioduszewski, A., \& Rupen, M. 2006, in VI Microquasar Workshop: Microquasars and Beyond, PoS (MQW6) 52

Dubois, R. 2006, in VI Microquasar Workshop: Microquasars and Beyond, PoS (MQW6) 68

Dubus, G. 2006a, A\&A, 451, 9

Dubus, G. 2006b, A\&A, 456, 801

Fargion, D., Konoplich, R. V., \& Salis, A. 1997, Z. Phys. C., 74, 571

Hartman, R. C., Bertsch, D. L., Bloom, S. D., et al. 1999, ApJS, 123, 79

Ho, C., \& Epstein, R. I. 1989, ApJ, 343, 277

Jones, F. C. 1968, Physical Review, 167, 1159

Manchester, R. N., Johnston, S., Lyne, A. G., et al. 1995, ApJ, 445, L137

Maraschi, L., \& Treves, A. 1981, MNRAS, 194, 1P

Martocchia, A., Motch, C., \& Negueruela, I. 2005, A\&A, 430, 245

Moderski, R., Sikora, M., Coppi, P. S., \& Aharonian, F. 2005, MNRAS, 363, 954

Paredes, J. M., Bosch-Ramon, V., \& Romero, G. E. 2006, A\&A, 451, 259

Paredes, J. M., Martí, J., Ribó, M., \& Massi, M. 2000, Science, 288, 2340

Sazonov, S. Y., \& Sunyaev, R. A. 2000, A\&A, 354, L53

Tavani, M., Arons, J., \& Kaspi, V. M. 1994, ApJ, 433, L37 